BMJ Open

Sport \&

Exercise

Medicine

\section{Is there an association between metabolic syndrome and rotator cuff- related shoulder pain? A systematic review}

To cite: Burne G, Mansfield M, Gaida JE, et al. Is there an association between metabolic syndrome and rotator cuffrelated shoulder pain? A systematic review. BMJ Open Sport \& Exercise Medicine 2019;5:e000544. doi:10.1136/ bmjsem-2019-000544

Accepted 3 November 2019

Check for updates

(C) Author(s) (or their employer(s)) 2019. Re-use permitted under CC BY-NC. No commercial re-use. See rights and permissions. Published by BMJ.

${ }^{1}$ Physiotherapy Department, Guy's and Saint Thomas' NHS Foundation Trust, London, UK ${ }^{2}$ Physiotherapy, University of Hertfordshire School of Health and Social Work, Hatfield, UK ${ }^{3}$ Allied Health Sciences, School of Health and Social Care, London Southbank University, London, UK

${ }^{4}$ Research Institute for Sport and Exercise (UCRISE), University of Canberra, Canberra, Australian Capital Territory, Australia ${ }^{5}$ School of Health and Social Work, University of Hertfordshire, Hatfield, UK

${ }^{6}$ Therapy Department, Central London Community Healthcare National Health Service Trust, London, UK

${ }^{7}$ Department of Physical Therapy and Rehabilitation Science, College of Health Sciences, Qatar University, Doha, Qatar

Correspondence to Graham Burne; graham.burne@gstt.nhs.uk

\section{ABSTRACT}

Objectives Rotator cuff-related shoulder pain (RCRSP) is a common upper limb complaint. It has been suggested that this condition is more common among people with cardiometabolic risk factors. This systematic review has synthesised evidence from case-control, cross-sectional and cohort studies on the association between metabolic syndrome (MetS) and RCRSP.

Design and data sources Five medical databases (MEDLINE, EMBASE, SCOPUS, CINAHL and AMED) and reference checking methods were used to identify all relevant English articles that considered MetS and RCRSP. Studies were appraised using the Newcastle-Ottawa Scale (NOS). Two reviewers performed critical appraisal and data extraction. Narrative synthesis was performed via content analysis of statistically significant associations.

Results Three cross-sectional, two case-control and one cohort study met the inclusion criteria, providing a total of 1187 individuals with RCRSP. Heterogeneity in methodology and RCRSP or MetS definition precluded a meaningful meta-analysis. Four of the included studies identified associations between the prevalence of MetS and RCRSP. Studies consistently identified independent cardiometabolic risk factors associated with RCRSP. All studies were level III evidence.

Summary and conclusion The low-moderate quality evidence included in this review suggests an association between MetS and RCRSP. Most studies demonstrated moderate quality on appraisal. The direction of association and cardiometabolic factors influencing should be investigated by longitudinal and treatment studies. These preliminary conclusions and clinical utility should be treated with caution due to limitations of the evidence base.

\section{INTRODUCTION}

Metabolic syndrome (MetS) is a complex disorder with high socioeconomic cost and mortality. ${ }^{12}$ It is a cluster of interconnected physiological, biochemical and clinical factors that is associated with cardiovascular disease (CVD) and type II diabetes mellitus (DM) risk. ${ }^{3}$ MetS has been associated with depression, ${ }^{4}$ cancer, ${ }^{5}$ health-related quality

\section{What is already known on this topic}

- The prevalence of metabolic syndrome (MetS) is reported to be $23 \%-46 \%$ in the general population.

- MetS has been associated with depression, cancer risk, low health-related quality of life, musculoskeletal pain (low back, neck and knee osteoarthritis) and all-cause mortality.

- Adipose tissue secretes a variety of bioactive molecules that individually or collectively suppress or enhance inflammation.

\section{What this study adds}

There is low-moderate quality evidence for an association between MetS and rotator cuff-related shoulder pain (RCRSP).

- While causality has not been established, associations between low-grade inflammatory biomarkers and RCRSP have been demonstrated in two included studies.

- It remains to be investigated if the impact of MetS exceeds the sum of all its individual risk factors (central obesity, dyslipidaemia, hypertension and insulin resistance).

- People with MetS are potentially at higher risk of shoulder pain, rotator cuff tears and more severe rotator cuff tears.

of life $^{6}$ and all-cause mortality. ${ }^{7}$ MetS has five primarily components-central obesity, elevated triglycerides, hypertension, low highdensity lipoprotein cholesterol (HDL-C) and elevated fasting glucose. This presentation is a manifestation of underlying cellular dysfunction, systemic inflammation and oxidative stress. $^{8-13}$

Chronic low-grade systemic inflammation is associated with MetS risk factors including, hypertension, type II DM, obesity and dyslipidaemia. ${ }^{8}$ 14-16 Systemic lowgrade inflammation has key differences to a classic inflammatory response. ${ }^{17}$ Specifically, 
low-grade systemic inflammation is characterised by subtly elevated acute phase proteins and elevated levels of active inflammatory cytokines in tissues, ${ }^{14}{ }^{18-20}$ with very low presence of neutrophils associated with acute inflammation. ${ }^{21}$

Excess adiposity is central to MetS. Diagnostic criteria typically mandate the presence of excess adiposity plus two of the other components (elevated triglycerides, hypertension, low HDL-C and elevated fasting glucose) ${ }^{22}$ Adipose tissue is metabolically active and secretes proinflammatory cytokines and proteinoids, which individually or collectively interact with various biological processes that suppress or enhance inflammation. ${ }^{23-25}$ This contributes to the development of many systemic complications, including abnormal insulin action. ${ }^{12} 2627$ Free fatty acid mobilisation is accelerated in the presence of insulin resistance leading to increased glucose production and dyslipidaemia, which perpetuates the low-grade systemic inflammation. ${ }^{8}$

Concordant with the rise in MetS, there is a global increase in the prevalence of musculoskeletal (MSK) diseases and disorders. ${ }^{28}$ Cardiometabolic risk factors and MSK pain are common comorbidities that feasibly share similar aetiology. ${ }^{29}$ A recent systematic review identified a relationship between MetS and Achilles tendinopathy. ${ }^{30}$ Furthermore, MetS is more prevalent in people with neck pain, ${ }^{31}$ low back pain ${ }^{32}{ }^{33}$ and knee osteoarthritis. ${ }^{34}$ One study showed that MetS was not associated with adhesive capsulitis, although associations with type II DM and hypertension were identified. ${ }^{35}$

Shoulder pain is a common MSK complaint in clinical practice with point prevalence ranging between $7 \%$ and $26 \% .^{36}{ }^{37}$ Symptoms associated with the rotator cuff and related tissues have been defined as rotator cuff-related shoulder pain (RCRSP). ${ }^{38}$ There is a growing body of research associating RCRSP with cardiometabolic risk factors, such as obesity, body mass index (BMI) and body fat. ${ }^{39-48}$ Conversely, other studies have reported no relationship with obesity and RCRSP. ${ }^{49-52}$ In a cross-sectional study, Miranda et a $\tilde{p}^{\tilde{3}}$ reported no association with obesity; however, people with type I DM had increased risk of RCRSP (OR 8.8; 95\% CI 1.9 to 40.3). A recent metaanalysis reported the prevalence of tendinopathy, and tendon thickness is increased in people with diabetes. ${ }^{54}$ Diabetes is associated with an upregulation of proinflammatory cytokines as the anti-inflammatory actions of insulin is impaired, inducing deregulation of the tendon matrix, which may lead to symptoms and functional impairments of the affected tendon. ${ }^{55}$ The role of dyslipidaemia association is inconsistent with two cohort studies and one cross-sectional study reporting elevated triglyceride and low HDL-C, impact pain and outcome in rotator cuff tears as compared with control groups. ${ }^{47657}$ Conversely, Longo et a $\bar{l}^{58}$ conducted a case-control study finding no significant difference between total cholesterol and triglyceride concentrations in people with rotator cuff tears and asymptomatic controls. Overall, systemic metabolic stress appears to promote low-grade inflammation, altered lipid metabolism and insulin resistance that may be associated with disease risk and/or progression of RCRSP. ${ }^{59}$

As the association between MetS and RCRSP is unclear, the aim of this study is to systematically review casecontrol, cross-sectional and cohort studies investigating the association between MetS and RCRSP.

\section{METHODS}

This review was conducted according to methodology guidelines (Centre for Reviews and Dissemination, 2009), and reported in accordance with the Preferred Reporting Items for Systematic Review and Meta-Analysis (PRISMA) statement. ${ }^{60}$ The review was based on an a priori protocol, which described the procedures that would be followed (eg, PICO, comprehensive search strategy and a piloted data extraction pro forma).

\section{Eligibility criteria}

All observational designs such as cross-sectional studies, case-control studies and cohort studies were included. The inclusion criteria were; (1) human study sample comprised of adult participants (>18 years), (2) study sample presented with signs and symptoms suggestive of RCRSP, (3) radiological findings recorded but not considered diagnostic of RCRSP, (4) MetS was recorded in medical history or met diagnostic criteria of three clinical risk factors and (5) participants recruited from specialist clinics were deemed to have accurate diagnosis of RCRSP or MetS (ie, based on clinical expertise, those participants recruited from a metabolic clinic were deemed to have MetS and participants attending an orthopaedic clinic for RCRSP).

Studies were excluded where a spinal source of pain was likely (eg, manikins indicating symptoms over neck and upper back) or the study sample comprised of participants with other pathologies (eg, fracture, dislocation, osteoarthritis, frozen shoulder, neurological presentations).

\section{Data sources and search strategy}

The search strategy and inclusion criteria were specified in advance. Studies were identified by an electronic search of MEDLINE, CINAHL, EMBASE, SCOPUS and AMED from inception to 20 June 2019. Additionally, reference lists of all included manuscripts were searched for relevant studies not identified in search strategy, combined with an unpublished (grey) literature search. Studies that included symptoms suggestive of RCRSP diagnosis and cardiometabolic variables related to MetS criteria were sought. Search terms were informed by a feasibility search and consultation with two health information librarians experienced in systematic review methodology. Example of the search terms and keywords used with MEDLINE (MeSH terms, Medical Subject Headings) are detailed in table 1. Records were imported into referencing software (Endnote X7), and duplicates removed. Based on eligibility criteria, the titles and abstracts were independently 


\section{Search terms}

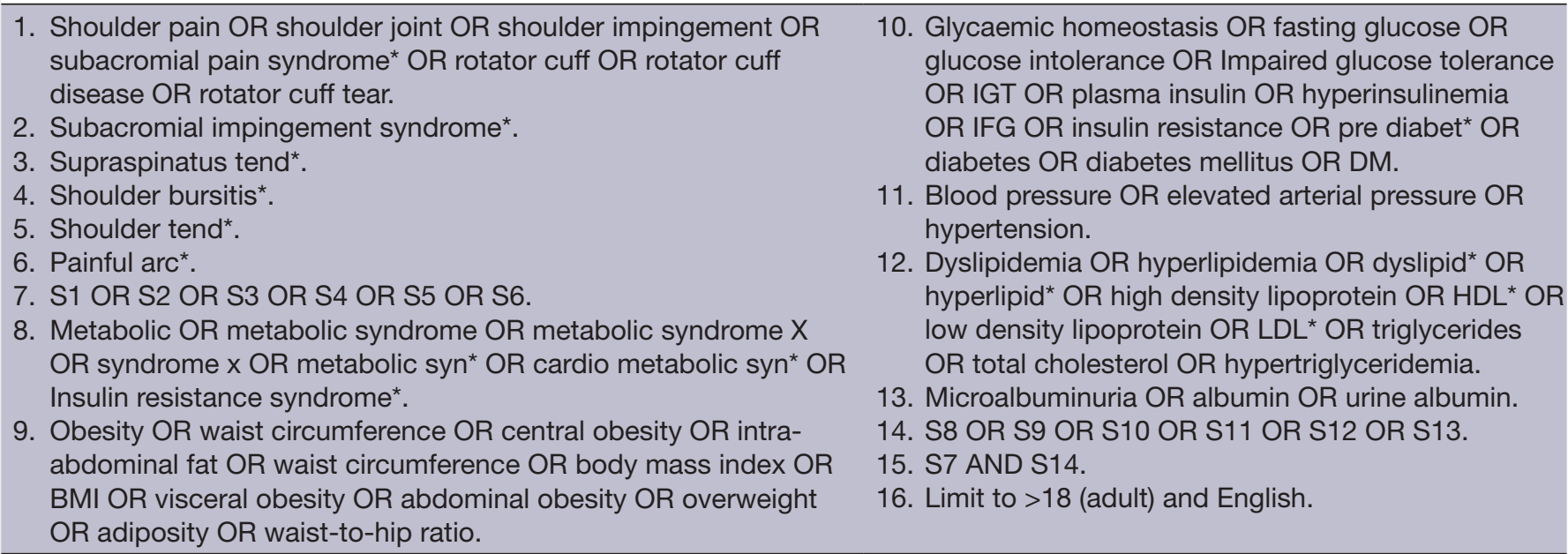

*Truncation command

reviewed by one reviewer $(\mathrm{GB})$ and relevant studies were accessed for full text. All potentially relevant studies were reviewed to determine final study selection (GB, MM); the reasons for exclusion at this stage were documented. A third reviewer (JL) was available for consultation and consensus.

\section{Data extraction}

Data extraction was performed by one reviewer (GB) and checked for verification and accuracy by a second reviewer (MM) using a predesigned piloted form. Data extraction for each study included: study location, study design, sample characteristics (age, gender), case definition and method of diagnosis for RCRSP, definition used to classify MetS or cardiometabolic risk factors included that met MetS definition within study population. Outcomes in terms of OR (unadjusted or adjusted), 95\% CIs and when available significant $\mathrm{p}$ values $<0.05$ were extracted. In circumstances where it was not possible to extract these data from the manuscript, corresponding authors were contacted to seek clarification or request individual patient data.

\section{Data analysis}

The included studies were assessed by two reviewers (GB, MM) through examination of the data extraction table. This demonstrated significant heterogeneity of subject characteristics (definition of RCRSP), cointerventions, exposure and criteria for diagnosing MetS, subsequently meta-analysis was precluded. A narrative synthesis with emphasis on study design and quality was undertaken as per the method described by Popay et al. Oxford Centre for Evidence-based Medicine (OCEBM) levels of evidence was used as guidance to evaluate selected articles. ${ }^{61}$

\section{Quality appraisal}

Quality of the included studies was evaluated using the Newcastle-Ottawa Scale (NOS) for cross-sectional, case-control and cohort studies. ${ }^{62}$ This appraisal tool is recommended by the Cochrane Handbook for Systematic Reviews of Interventions. ${ }^{63}$ The NOS checklist assesses quality of articles across three domains: selection of the study groups; comparability of the groups and control for cofounding factors; and exposure. The minimum and maximum scores available is 0 and 9 , respectively. The studies with score $\geq 6$; $\geq 3$ score $<6$; and score $<3$ were considered high quality, moderate quality and low quality, respectively. ${ }^{64}$ Two reviewers (GB, MM) independently examined the studies for risk of bias and internal validity (table 2 ). In order to assess interobserver agreement of the risk of bias, the kappa coefficient $(\kappa)$ was used. ${ }^{65}$

\section{RESULTS}

\section{Search strategy}

Figure 1 shows search and study selection process. After exclusions, 36 studies required the full text to be assessed. Out of these, 30 studies were excluded (23 studies had incomplete cardiometabolic details available to establish a MetS definition, 5 studies had no shoulder pain data and 2 studies did not meet participant criteria), resulting in 6 studies $^{42} 435566-68$ for inclusion in this review.

\section{Study characteristics}

Table 3 presents a summary of characteristics and findings of each included study. Within the construct of RCRSP; four studies examined people diagnosed with symptomatic rotator cuff tendinopathy and two studies examined people with symptomatic rotator cuff tears (partial and full thickness). Four studies were cross-sectional, one case controlled and one retrospective cohort. The total number of individuals across the studies was 8259 (mean age 50.9 years), of which 1187 were people experiencing RCRSP. Five studies recruited participants from health centres or hospitals. ${ }^{42} 43556768$ Applegate et $a t^{66}$ 


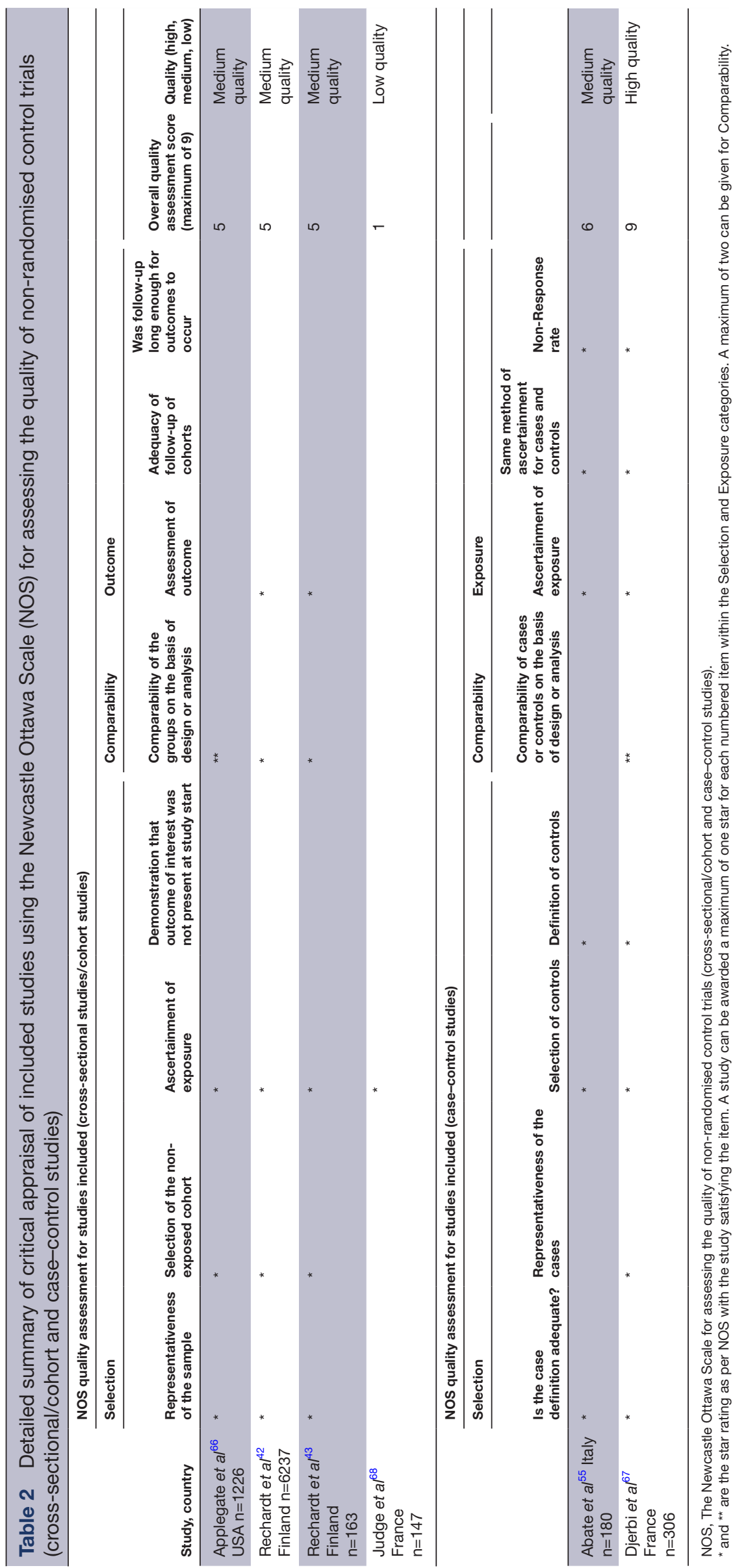




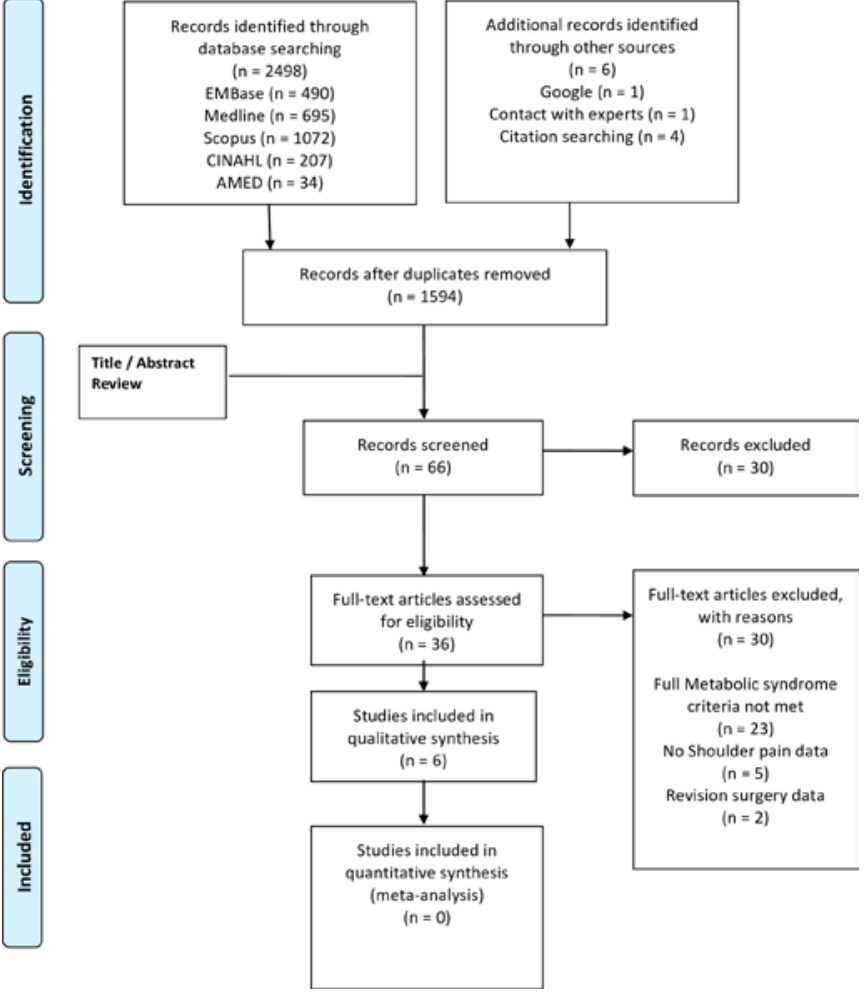

Figure 1 PRISMA flow diagram showing study selection process. ${ }^{60}$ PRISMA, Preferred Reporting Items for Systematic Review and Meta-Analysis.

recruited participants from 17 diverse production facilities, including food processing, manufacturing, assembly lines and office jobs.

\section{Quality assessment}

The six included studies were clinical human reports and considered level III evidence in line with OCBEM guidance. The results of the quality appraisal are shown in table 2. According to our definition, $1 / 6$ of the studies were good quality, 4/6 medium quality and $1 / 6$ of low quality. This was due to a number of common deficiencies in the methodological quality of these studies. With the exception of one study, ${ }^{67}$ the others did not report a study protocol. Response rates from preliminary sampled subjects were not reported in three studies, ${ }^{42} 4366$ thus substantial selection bias may have occurred. Studies tended to report variables for all their stated aims but did not specify which aims were determined in advance of conducting the study. Study quality as assessed by the NOS varied considerably across the studies ranging from $1 / 9$ to $9 / 9$, the main issues being lack of adequate diagnosis, definition of controls and lack of adjustment for potential cofounders (table 2). Interobserver agreement regarding the risk of bias was considered 'substantial' $(\kappa=0.76)$ according to the Viera and Garrett ${ }^{69}$ kappa interpretation model.

\section{RCRSP diagnosis classification}

The six studies had inconsistent diagnostic classification of RCRSP through a mix of self-reported symptoms, clinical findings and radiological imaging (table 3). For example, one study confirmed RCRSP as pain during functional activities (range of motion) and sonographic diagnosis of a partial or full thickness rotator cuff tear. ${ }^{55}$ Conversely, one study utilised the international classification of disease (ICD) 10 code $^{70}$ and one other study utilised radiography to differentiate primary or centred osteoarthritis. ${ }^{67}$

\section{MetS diagnosis classification}

MetS diagnosis was clearly defined within two studies using the National Cholesterol Education Programme Adult Treatment Panel III (NCEP ATP III). ${ }^{42} 43$ The remaining studies did not state which definition criteria was used (table 3). Within data extraction all studies met a definition set by many international expert groups, such as the WHO and the International Diabetes Federation (IDF). There is no currently agreed diagnostic consensus. ${ }^{8}$ The variance is attributed to the focus of each definition, from the obesity-centric IDF to a glucocentric WHO definition and the collection of statistically elevated CVD risk factor by the NCEP ATP III.

\section{Analysis}

Of the studies included, four studies reported a positive association between MetS and shoulder pain. Applegate et $a l^{66}$ reported an adjusted OR for glenohumeral joint pain among people with the highest CVD risk score (18+), scaled in accordance with a modified Framingham Heart Study's risk assessment, ${ }^{71}$ namely, hypertension, dyslipidaemia and diabetes of 4.55 (95\% CI 1.99 to 10.40 , $\mathrm{p}<0.001)$. Similarly, the adjusted OR for RC tendinopathy in those with the highest CVD risk score was 5.97 (95\% CI 2.12 to $16.83, \mathrm{p}<0.008)$. Djerbi et a ${ }^{67}$ reported an unadjusted OR for symptomatic rotator cuff tears (SCOI 1-3) of OR 2.55 (95\% CI 1.4 to $4.58, \mathrm{p}=0.0017$ ) for obesity, $2.04(95 \%$ CI 1.18 to $3.52, \mathrm{p}=0.0102)$ for systolic $\mathrm{BP}$ and 7.69 (95\% CI 3.35 to 17.25 , $\mathrm{p}<0.0001$ ) for dyslipidaemia. Higher grade tears (SCOI 4) were also associated with obesity (OR 2.105, $\mathrm{p}=0.0117$ ), systolic BP (OR 4.311, $\mathrm{p}<0.0001$ ) and dyslipidaemia (OR 2.867, $\mathrm{p}=0.0004$ ). Although, when adjusted, only dyslipidaemia (OR 4.920; 95\% CI 2.046 to $11.834, \mathrm{p}=0.0004)$ and systolic BP (OR $3.215 ; 95 \%$ CI 1.67 to $6.19, \mathrm{p}=0.0005$ ) were associated. Rechardt $e t a t^{42}$ reported an adjusted OR for unilateral glenohumeral joint pain over a 3 month period of 1.7 (95\% CI 1.3 to 2.1) for MetS (NCEP ATP II definition) in males. This study found MetS was not associated with rotator cuff tendinopathy (OR $0.7 ; 95 \%$ CI 0.4 to 1.1 ). Juge et al reported a significant difference in the proportion of people with established MetS criteria (obesity, DM, dyslipidaemia, hypertension) between RCRSP (6.5\%) and those with osteoarthritis $(0 \%, \mathrm{p}=0.03)$. Two studies reported no association between MetS and RCRSP. Abate et $a \tilde{l}^{5}$ reported no significant difference in rotator cuff tear prevalence in people with cardiometabolic risk factors meeting MetS criteria. Although, this study did report positive power of the predictor coefficient $(\beta)$ for 


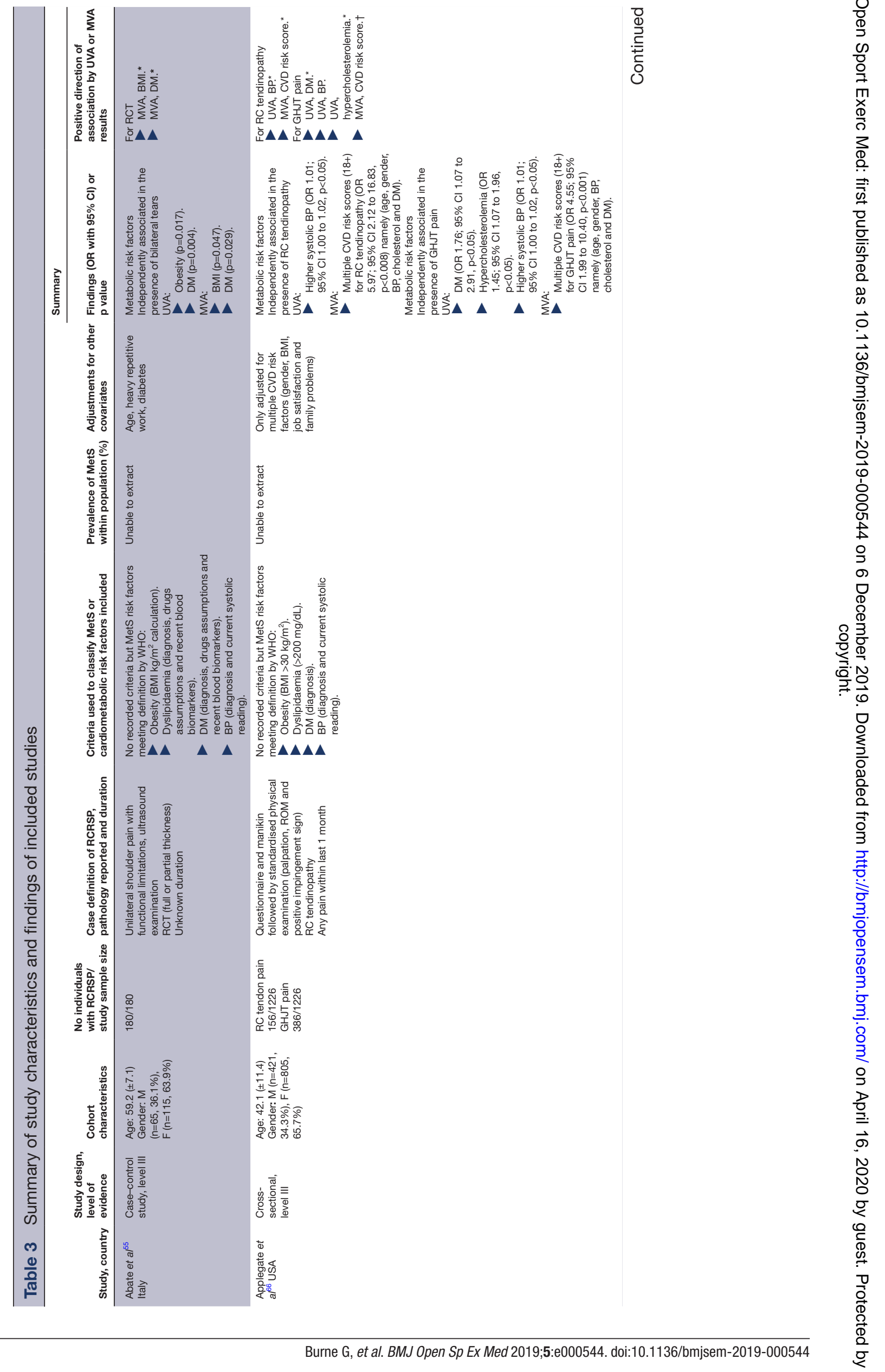




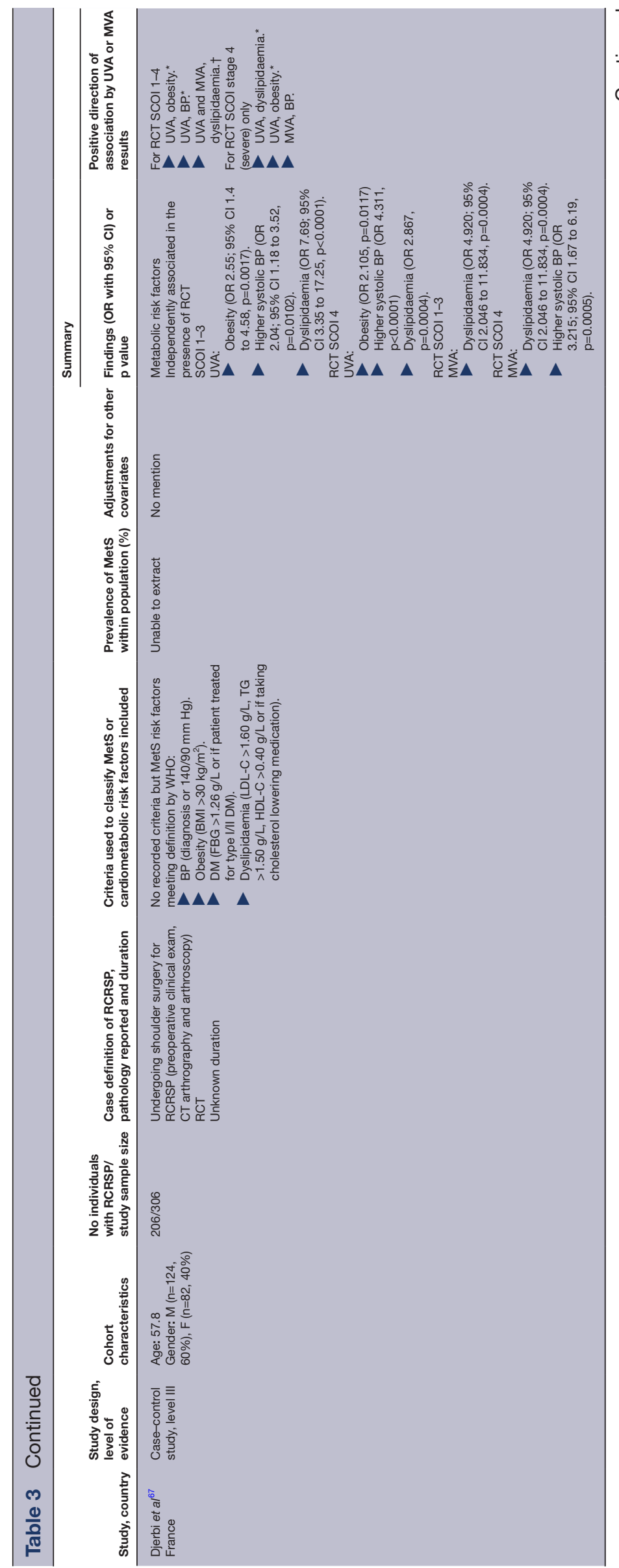




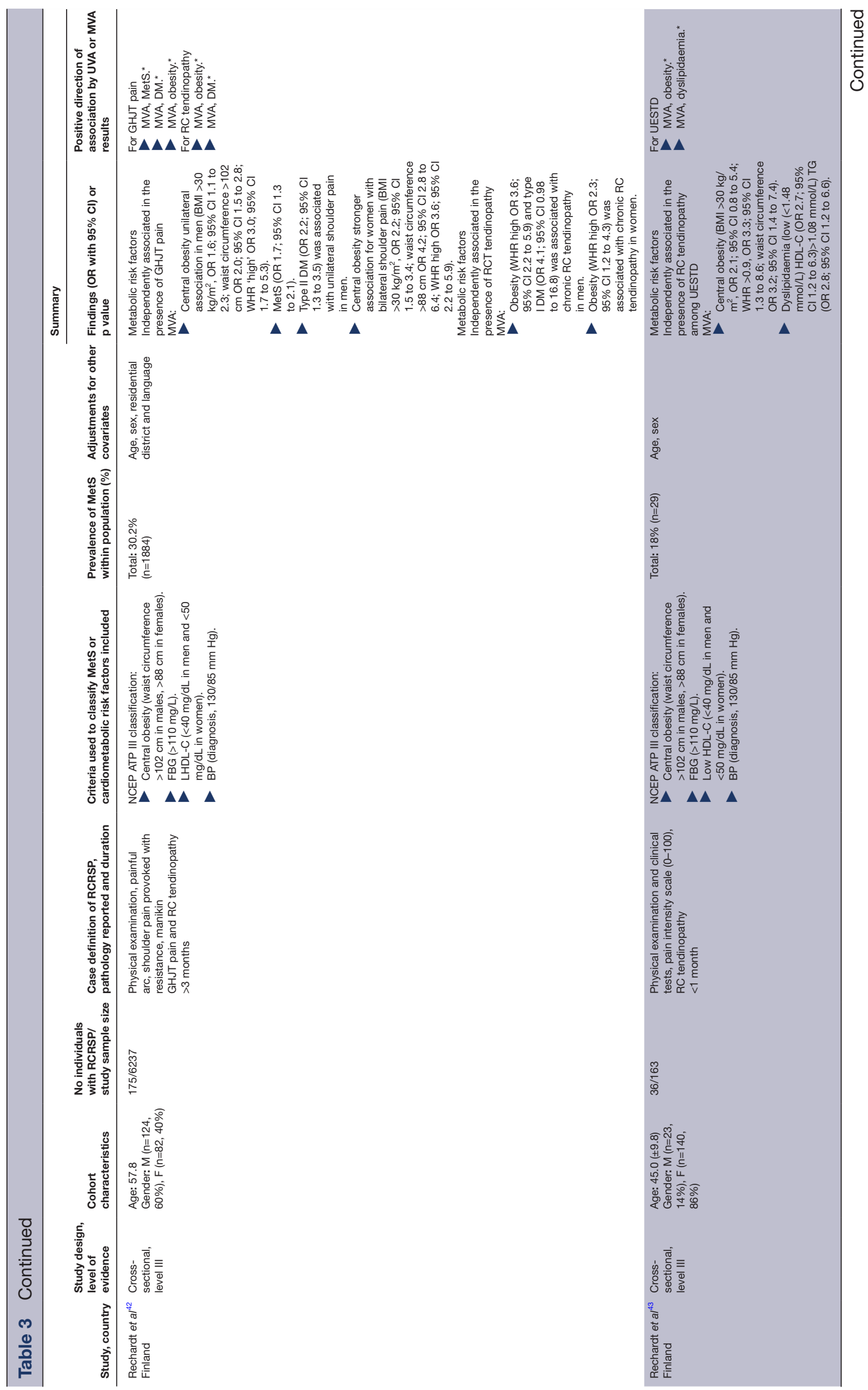




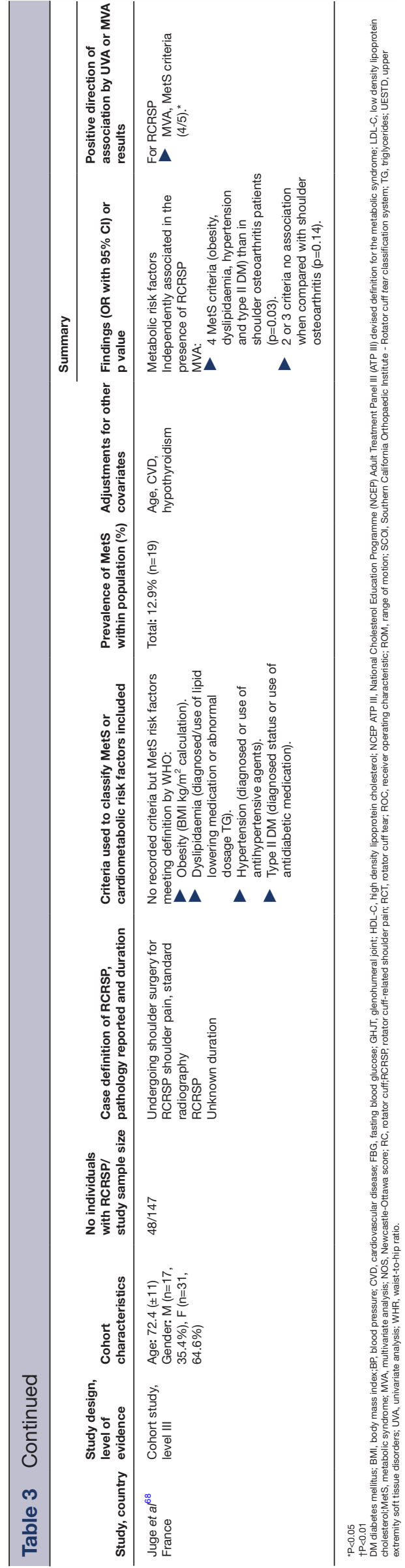

cardiometabolic risk factors of BMI $(\beta=0.32, \mathrm{p}=0.047)$ and DM $(\beta=1.01, p=0.029) .{ }^{55}$ Indicating higher values of $\mathrm{BMI}$ and DM are related to a higher probability to detect rotator cuff tears. Similarly, Rechardt et at ${ }^{43}$ reported no association with pain intensity in RCRSP and MetS, however, did report central obesity (waist-to-hip ratio $>0.9$, OR $3.3 ; 95 \%$ CI 1.3 to 8.6 ; waist circumference OR 3.2; $95 \%$ CI 1.4 to 7.4 ) and dyslipidaemia (OR 2.7; $95 \%$ CI 1.2 to 6.3 ) were positively associated when adjusting for age and sex.

\section{DISCUSSION}

The low-moderate quality evidence included in this review suggests an association between MetS and RCRSP. From the six studies included (one good quality, one low quality and four medium quality) it appears there is an association between MetS (and its constituent factors) and RCRSP. Two medium quality studies reported no association with MetS, but reported associations between cardiometabolic risk factors (BMI, DM and dyslipidaemia) and painful shoulders with identifiable unilateral rotator cuff tears and with RCRSP. In addition, populations with independent cardiometabolic risk factors associated with RCRSP were identified across all studies (table 3); age $>45$ years, obesity (notably central adiposity), DM, hypertension and dyslipidaemia.

Obesity, DM, hypertension and dyslipidaemia are components of MetS and are also interrelated. It is therefore difficult to separate the individual versus combined effect of these factors. Previous research on RCRSP has identified relationships with independent cardiometabolic risk factors including adiposity, ${ }^{41} 4546$ diabetes, ${ }^{54} 72$ dyslipidaemia $^{57}$ and hypertension. ${ }^{73}$ For example, an 11-year nation-wide, longitudinal follow-up study of 498678 people of whom 26664 developed RCRSP showed a diagnosis of diabetes had a hazard ratio (HR) of 1.47 (95\% CI 1.41 to 1.54; $\mathrm{p}<0.0001$ ) for RCRSP and hyperlipidaemia had a HR of 1.48 (95\% CI 1.42 to 1.55 ; $\mathrm{p}<0.0001)$ for RCRSP. ${ }^{74}$ Similarly, a 4-year, longitudinal follow-up of 10044 people with clustered back, neck and/or shoulder pain showing a diagnosis of diabetes had a relative risk of 1.64 (95\% CI 1.23 to 2.18) for shoulder pain in males and hyperlipidaemia had a relative risk of 1.19 (95\% CI 0.98 to 1.44) for shoulder pain in males. ${ }^{75}$ Furthermore, a large case-control study of 5000 patients with RCRSP identified that increased BMI (BMI between 25.1 and 30) was independently associated (OR 1.15 ; $95 \%$ CI 1.02 to 1.31 ) with RCRSP. ${ }^{76}$ Given these associations, RCRSP and cardiometabolic risk factors may share a common aetiology. As yet it is unclear whether the impact of MetS of RCRSP exceeds the sum of all its individual factors. More studies are required to assess the independent contribution of MetS; however, the above aggregative risk factors suggest MetS may play a role in RCRSP.

The postulated pathophysiological mechanisms cannot be fully understood on the basis of available studies. While causality has not been established, associations between low-grade inflammatory biomarkers and RCRSP have been shown. Excess central adiposity is key 
constituent of MetS and adipose tissue is a rich source of circulating proinflammatory cytokines; collectively referred to as adipocytokines, including, tumour necrosis factor alpha (TNF- $\alpha)$, IL-1 $\beta$, IL-1, IL-6, adiponectin, leptin and restinin. Increased cytokine expression has been observed in the subacromial bursa (IL-1 and IL-6) and partial thickness rotator cuff tear tissue (IL-6) from subjects undergoing rotator cuff surgery. ${ }^{77-79}$ Also, increased expression serum levels of TNF- $\alpha$ within subacromial bursa specimens have been retrieved during symptomatic rotator cuff tendon surgery. ${ }^{80}$ In parallel, reduced level of IL-10 is detected in patients with obesity, dyslipidaemia and insulin resistance. ${ }^{81}$ IL-10 is an antiinflammatory cytokine that modulates the immune system by antagonising the activities of proinflammatory cytokines. ${ }^{12}$ It is possible that the resultant increased proinflammatory adipocytokine expression and antiinflammatory adipocytokine suppression is a risk factor for developing RCRSP. ${ }^{43}$

Dysregulation of adipokines resulting from central obesity contributes to DM. ${ }^{82}$ Type I and type II DM has been associated with increased risk of chronic RCRSP. ${ }^{2354}$ Hyperglycaemia has vasodilatory and proinflammatory actions mediated by cytokine suppression. ${ }^{83}$ Increased glucose availability had direct effects on tendons of the rotator cuff and can alter the physiological behaviour of tendons. ${ }^{84}$ The low-grade, subclinical, but persistent inflammation in DM has been shown to affect collagen crosslinking, proteoglycan content, cytokine activity, which can lead to tendon thickening and matrix degeneration. ${ }^{23} 5485$

Multifunctional (proinflammatory or antiinflammatory) proteins such as leptin, adiponectin, resitin and vistafin have been discovered within the adipocytokine family. These are involved with the lowgrade inflammation encountered in obese and diabetic subjects linked to the pathogenesis of MetS. ${ }^{86}{ }^{87}$ Little is known about their role within MSK disorders. An association has been reported between increased proinflammatory leptin and pain related to arthrosis of the shoulder. ${ }^{88}$ Decreased anti-inflammatory adiponectin levels have been associated to pathological conditions of diabetes and MetS ${ }^{89}$ and found to be lower within the synovial fluid of people with shoulder pain. ${ }^{88}$ Although little is known about the role of these proteins they may have a role within shoulder pain and metabolic disorders.

There is inconsistency regarding the link between hypercholesterolemia and RCRSP. Longo et $a p^{58}$ found no association between elevated total cholesterol and rotator cuff injury; however, the mean triglyceride concentration was below the threshold $1.7 \mathrm{mmol} / \mathrm{L}$ for $62.5 \%$ (mean $1.49 \mathrm{mmol} / \mathrm{L}$ ) to meet the diagnostic criteria required for MetS. In contrast, Abboud et a $\tilde{p}^{7}$ did find a correlation between rotator cuff tears and increased cholesterol levels, specifically with elevated low-density lipoprotein cholesterol (LDL-C) and low HDL-C levels. The mechanism by which dyslipidaemia affects shoulder pain is also unknown. It is hypothesised that due to catabolism of HDL-C increased LDL-C could initiate and maintain, low-grade persistent inflammation within the rotator cuff tendon, as shown in vivo studies on rotator cuff tendon in mice. ${ }^{2390}$ There may also be direct cholesterol deposition within tendon. ${ }^{91-95}$ Dyslipidaemia is a known precursor for hypertension and CVD. ${ }^{96}$ This may also contribute to RCRSP, as subsequent endothelial damage caused by hypertension and deregulated lipid metabolism has been associated with tissues damage in tendons. ${ }^{97}$ This is supported by Gumina et $a l^{33}$ who noted hypertension was associated with a twofold increase of a large rotator cuff tear (OR 2.09; 95\% CI 1.39 to 3.16) and fourfold increase of massive rotator cuff tear (OR 4.30; $95 \%$ CI 2.44 to 7.58 ). However, the study was not able to adjust for the range of antihypertensive medication drugs, thus not possible to define this influence on tear dimensions. This tissue damage in the presence of hypertension has been linked to hypovascularity and hypoxia, ultimately depriving tissues of appropriate nutrient exchange. ${ }^{98}$ This triggered deregulation of the tendon matrix may lead to symptoms and functional impairments of the affected tendon in RCRSP. ${ }^{55}$

These findings should be viewed with some caution as biological measures do not work in isolation but rather interact with an array of complex systems and consideration of a composite of measures reflecting system functioning. ${ }^{99}$ No study has reported biomarkers that might represent potential mediators between MetS and RCRSP. ${ }^{100}$ Furthermore, the evidence supporting the influence of postulated MetS-induced inflammatory biomarkers and its association with symptoms in either the acute or chronic stage of RCRSP is lacking. ${ }^{100}$ RCRSP has a multifactorial aetiology. It is recommended these findings be considered within a wider management of RCRSP including recognition of increased excitability of the peripheral and central neuraxis or decreased inhibition, ${ }^{101}$ psychosocial and work-related factors. ${ }^{101-103}$ Clinicians should, however, remain cognisant of cardiometabolic status throughout rehabilitation, particularly in respect to how metabolic stress may precipitate low-grade systemic inflammation ${ }^{17}$ and it's purported deleterious impact in RCRSP.

\section{Limitations}

This review summarises the potential association between MetS and RCRSP, the basis of which are limited to the outcomes used by the original studies. With no clear consensus across the included literature, the clinical utility of identifying MetS in patients with RCRSP may be questioned.

The diagnostic criteria for RCRSP varied considerably across studies. Including a combination of participant selfreported symptoms, clinical assessment testing and varied radiological imaging techniques, which may impact the reliability and external validity of findings. Furthermore, four studies included an assembled MetS profile, with only two studies stated predetermined criteria used to define MetS. Currently, no universally agreed diagnostic 
criteria for MetS or RCRSP exists, which can be used as a comparative within both clinical and research fields. It is recognised that the classification of the NOS scoring criteria ('high', 'moderate' and 'low') adopted in this review is novel and has limited evidence. However, this method of 'grading' can provide context when applying our findings to future research and clinical practice. The standardisation of MetS and RCRSP diagnosis criteria is essential for clinicians and researchers alike to generate research. This allows results to be compared and pooled to make meaningful conclusions regarding metabolic factors in MSK shoulder pain. It is important to consider methodological quality of the included studies as the majority were cross-sectional in which selection bias cannot be avoided. For example, associations maybe accentuated because the investigators accessed pools of RCRSP patients from specialist health centres with access to pools of MetS subjects. Furthermore, it cannot be determined that exposure predetermined disease since data were ascertained at the same time. Overall, the available evidence was of relatively low-moderate quality, specifically, all studies were of level III evidence. With evident methodological concerns, as described above, it is likely the findings from this review will evolve as highquality studies become available.

\section{Future research}

Questions remain on the direction of RCRSP association and therefore on the possibility of an effective management of MetS and vice versa. Initially, researchers should address the need for harmonising the diagnostic criteria for MetS, including the definition of optimal metabolic biomarkers and normative age-related values. The consistency of RCRSP diagnostic criteria also needs to be established in future studies; this will enhance the analysis of results when this review is updated. Cross-sectional design limits causal inference. Well-controlled prospective large-scale studies will be an important next step to examining the relationship between MetS and RCRSP. This should include examining changes in pain, cofounders, health behaviours and biological measures in order to better appreciate potential causal direction. When sufficient homogenous representative samples are available, a repeated systematic review with meta-analysis should be considered to pool data of an adequate sample size to quantify the strength of the association. This may then allow investigations into the effects comorbidity management could influence RCRSP associated symptoms and through which biological pathway they act. Furthermore, this may propose a subcategory of metabolic shoulder pain introducing new ideas of management and prognosis.

\section{CONCLUSIONS}

The results of this review suggest a positive association between MetS and RCRSP in six low-moderate quality studies. Specifically, patients with MetS are potentially at higher risk of shoulder pain, rotator cuff tears and more severe rotator cuff tears. While causality has not been established, two studies included in this review have associations between low-grade inflammatory biomarkers and RCRSP.

These preliminary conclusions should be treated with caution due to significant methodological limitations and concerns regarding their validity. It is likely that future high-quality primary studies may challenge these preliminary conclusions.

Twitter Graham Burne @GrahamBurneWHL, Michael Mansfield @MM_Physio, Jamie E Gaida @tendonresearch and Jeremy S Lewis @JeremyLewisPT

Acknowledgements Guy's and St Thomas' NHS Foundation Trust-This project was completed with the support and funding of my employer. Particular thanks I share with Chris Tack for his time, shared experience and direction through the discussion.

Contributors GB: protocol design, primary investigator and author. MM: inclusion/ exclusion reviewer, secondary data extraction reviewer and critical appraiser (risk of bias assessment), editing of the manuscript. JEG: guidance with the original protocol, editing of the manuscript. JSL: protocol design, dissertation supervisor, editing of the manuscript.

Funding This study was undertaken in partial fulfilment of an MSc Advanced Physiotherapy undertaken at the Department of Allied Health Professions and Midwifery, School of Health and Social Work, University of Hertfordshire.

Competing interests None declared.

Patient consent for publication Not required.

Provenance and peer review Not commissioned; externally peer reviewed.

Data availability statement All data relevant to the study are included in the article.

Open access This is an open access article distributed in accordance with the Creative Commons Attribution Non Commercial (CC BY-NC 4.0) license, which permits others to distribute, remix, adapt, build upon this work non-commercially, and license their derivative works on different terms, provided the original work is properly cited, appropriate credit is given, any changes made indicated, and the use is non-commercial. See: http://creativecommons.org/licenses/by-nc/4.0/.

ORCID iDs

Graham Burne http://orcid.org/0000-0001-8517-8913

Michael Mansfield http://orcid.org/0000-0003-4258-8247

Jamie E Gaida http://orcid.org/0000-0002-4504-9188

Jeremy S Lewis http://orcid.org/0000-0001-7870-9165

\section{REFERENCES}

1 O'Neill S, O'Driscoll L. Metabolic syndrome: a closer look at the growing epidemic and its associated pathologies. Obes Rev 2015;16:1-12

2 Scholze J, Alegria E, Ferri C, et al. Epidemiological and economic burden of metabolic syndrome and its consequences in patients with hypertension in Germany, Spain and Italy; a prevalence-based model. BMC Public Health 2010;10:529.

3 Mancia G, Bombelli M, Facchetti R, et al. Impact of different definitions of the metabolic syndrome on the prevalence of organ damage, cardiometabolic risk and cardiovascular events. $J$ Hypertens 2010;28:999-1006.

4 Shinkov A, Borissova A-M, Kovatcheva R, et al. Increased prevalence of depression and anxiety among subjects with metabolic syndrome and known type 2 diabetes mellitus - a population-based study. Postgrad Med 2018;130:251-7.

5 Esposito K, Chiodini P, Colao A, et al. Metabolic syndrome and risk of cancer: a systematic review and meta-analysis. Diabetes Care 2012;35:2402-11.

6 Ford ES, Li C, Syndrome M. Metabolic syndrome and healthrelated quality of life among U.S. adults. Ann Epidemiol 2008;18:165-71.

7 Mottillo S, Filion KB, Genest J, et al. The metabolic syndrome and cardiovascular risk a systematic review and meta-analysis. J Am Coll Cardiol 2010;56:1113-32. 
8 Kassi E, Pervanidou P, Kaltsas G, et al. Metabolic syndrome: definitions and controversies. BMC Med 2011;9:48.

9 Grundy SM. Metabolic syndrome pandemic. Arterioscler Thromb Vasc Biol 2008;28:629-36.

10 Shulman GI. Ectopic fat in insulin resistance, dyslipidemia, and cardiometabolic disease. N Engl J Med 2014;371:1131-41.

11 Abraham TM, Pedley A, Massaro JM, et al. Association between visceral and subcutaneous adipose depots and incident cardiovascular disease risk factors. Circulation 2015;132:1639-47.

12 Srikanthan K, Feyh A, Visweshwar H, et al. Systematic review of metabolic syndrome biomarkers: a panel for early detection, management, and risk stratification in the West Virginian population. Int J Med Sci 2016;13:25-38.

13 McCracken E, Monaghan M, Sreenivasan S. Pathophysiology of the metabolic syndrome. Clin Dermatol 2018;36:14-20.

14 León-Pedroza Jl, González-Tapia LA, del Olmo-Gil E, et al. LowGrade systemic inflammation and the development of metabolic diseases: from the molecular evidence to the clinical practice. Cirugía y Cirujanos 2015;83:543-51.

15 Esser N, Legrand-Poels S, Piette J, et al. Inflammation as a link between obesity, metabolic syndrome and type 2 diabetes. Diabetes Res Clin Pract 2014;105:141-50.

16 Monteiro R, Azevedo I. Chronic inflammation in obesity and the metabolic syndrome. Mediators Inflamm 2010;2010:289645

17 Medzhitov R. Origin and physiological roles of inflammation. Nature 2008;454:428-35.

18 Makki K, Froguel P, Wolowczuk I. Adipose tissue in obesityrelated inflammation and insulin resistance: cells, cytokines, and chemokines. ISRN Inflamm 2013;2013:1-12.

19 Singh AK, Kari JA. Metabolic syndrome and chronic kidney disease. Curr Opin Nephrol Hypertens 2013;22:198-203.

20 Odegaard JI, Chawla A. Pleiotropic actions of insulin resistance and inflammation in metabolic homeostasis. Science 2013;339:172-7.

21 Chatzigeorgiou A, Karalis KP, Bornstein SR, et al. Lymphocytes in obesity-related adipose tissue inflammation. Diabetologia 2012;55:2583-92.

22 Grundy SM, Cleeman JI, Daniels SR, et al. Diagnosis and management of the metabolic syndrome. Circulation 2005;112:2735-52.

23 Abate M, Schiavone C, Salini V, et al. Occurrence of tendon pathologies in metabolic disorders. Rheumatology 2013;52:599-608.

24 Pietrzak M. Adhesive capsulitis: an age related symptom of metabolic syndrome and chronic low-grade inflammation? Med Hypotheses 2016;88:12-17.

25 Rechardt M, Viikari-Juntura E, Shiri R. Adipokines as predictors of recovery from upper extremity soft tissue disorders. Rheumatology 2014;53:2238-42.

26 Hotamisligil GS, Shargill NS, Spiegelman BM. Adipose expression of tumor necrosis factor-alpha: direct role in obesity-linked insulin resistance. Science 1993;259:87-91.

27 Yudkin JS. Adipose tissue, insulin action and vascular disease: inflammatory signals. Int $J$ Obes 2003;27:S25-8.

28 Wearing SC, Hennig EM, Byrne NM, et al. Musculoskeletal disorders associated with obesity: a biomechanical perspective. Obes Rev 2006;7:239-50.

29 Dominick $\mathrm{CH}$, Blyth FM, Nicholas MK. Unpacking the burden: understanding the relationships between chronic pain and comorbidity in the general population. Pain 2012;153:293-304

30 Kozlovskaia M, Vlahovich N, Ashton KJ, et al. Biomedical risk factors of Achilles tendinopathy in physically active people: a systematic review. Sports Med Open 2017;3.

31 Mäntyselkä P, Kautiainen $\mathrm{H}$, Vanhala M. Prevalence of neck pain in subjects with metabolic syndrome--a cross-sectional populationbased study. BMC Musculoskelet Disord 2010;11:171.

32 Duruöz MT, Turan Y, Gürgan A, et al. Evaluation of metabolic syndrome in patients with chronic low back pain. Rheumatol Int 2012;32:663-7.

33 Tsuboi $\mathrm{Y}$, Ueda $\mathrm{Y}$, Sugimoto T, et al. Association between metabolic syndrome and disability due to low back pain among care workers. Int J Occup Med Environ Health 2018;31:165-72.

34 Niu J, Clancy M, Aliabadi P, et al. Metabolic syndrome, its components, and knee osteoarthritis: the Framingham osteoarthritis study. Arthritis Rheumatol 2017;69:1194-203.

35 Austin DC, Gans I, Park MJ, et al. The association of metabolic syndrome markers with adhesive capsulitis. J Shoulder Elbow Surg 2014;23:1043-51.

36 Silverstein B, Viikari-Juntura E, Kalat J. Use of a prevention index to identify industries at high risk for work-related musculoskeletal disorders of the neck, back, and upper extremity in Washington state, 1990-1998. Am J Ind Med 2002;41:149-69.
37 Luime JJ, Koes BW, Hendriksen IJM, et al. Prevalence and incidence of shoulder pain in the general population; a systematic review. Scand J Rheumatol 2004;33:73-81.

38 Lewis J. Rotator cuff related shoulder pain: assessment, management and uncertainties. Man Ther 2016;23:57-68.

39 Ertan S, Ayhan E, Güven MF, et al. Medium-Term natural history of subacromial impingement syndrome. J Shoulder Elbow Surg 2015;24:1512-8.

40 Gaida JE, Ashe MC, Bass SL, et al. Is adiposity an underrecognized risk factor for tendinopathy? A systematic review. Arthritis Rheum 2009;61:840-9.

41 Gumina S, Candela V, Passaretti D, et al. The association between body fat and rotator cuff tear: the influence on rotator cuff tear sizes. J Shoulder Elbow Surg 2014;23:1669-74.

42 Rechardt M, Shiri R, Karppinen J, et al. Lifestyle and metabolic factors in relation to shoulder pain and rotator cuff tendinitis: a population-based study. BMC Musculoskelet Disord 2010;11:165

43 Rechardt M, Shiri R, Lindholm H, et al. Associations of metabolic factors and adipokines with pain in incipient upper extremity soft tissue disorders: a cross-sectional study. BMJ Open 2013;3:e003036.

44 Koca TT, Arslan A, Özdemir F, et al. Clinical features of patients diagnosed with degenerative rotator cuff tendon disease: a 6-month prospective-definitive clinical study from turkey. J Phys Ther Sci 2017;29:1433-7.

45 Sansone V, Consonni O, Maiorano E, et al. Calcific tendinopathy of the rotator cuff: the correlation between pain and imaging features in symptomatic and asymptomatic female shoulders. Skeletal Radiol 2016;45:49-55.

46 Wendelboe AM, Hegmann KT, Gren LH, et al. Associations between body-mass index and surgery for rotator cuff tendinitis. $J$ Bone Joint Surg Am 2004;86:743-7.

47 Cancienne JM, Brockmeier SF, Rodeo SA, et al. Perioperative serum lipid status and statin use affect the revision surgery rate after arthroscopic rotator cuff repair. Am J Sports Med 2017:45:2948-54

48 Garcia GH, Liu JN, Wong A, et al. Hyperlipidemia increases the risk of retear after arthroscopic rotator cuff repair. J Shoulder Elbow Surg 2017;26:2086-90.

49 Bodin J, Ha C, Sérazin C, et al. Effects of individual and workrelated factors on incidence of shoulder pain in a large working population. J Occup Health 2012;54:278-88.

50 Roquelaure $\mathrm{Y}$, Bodin $\mathrm{J}, \mathrm{Ha} \mathrm{C}$, et al. Personal, biomechanical, and psychosocial risk factors for rotator cuff syndrome in a working population. Scand J Work Environ Health 2011;37:502-11.

51 Silverstein BA, Bao SS, Fan ZJ, et al. Rotator cuff syndrome: personal, work-related psychosocial and physical load factors. $J$ Occup Environ Med 2008;50:1062-76.

52 Viikari-Juntura E, Shiri R, Solovieva S, et al. Risk factors of atherosclerosis and shoulder pain - Is there an association? A systematic review. European Journal of Pain 2008;12:412-26.

53 Miranda H, Viikari-Juntura E, Heistaro S, et al. A population study on differences in the determinants of a specific shoulder disorder versus nonspecific shoulder pain without clinical findings. Am J Epidemiol 2005;161:847-55.

54 Ranger TA, Wong AMY, Cook JL, et al. Is there an association between tendinopathy and diabetes mellitus? A systematic review with meta-analysis. Br J Sports Med 2016;50:982-9.

55 Abate M, Di Carlo L, Salini V, et al. Risk factors associated to bilateral rotator cuff tears. Orthop Traumatol Surg Res 2017;103:841-5.

56 Lai J, Robbins CB, Miller BS, et al. The effect of lipid levels on patient-reported outcomes in patients with rotator cuff tears. JSES Open Access 2017;1:133-8.

57 Abboud JA, Kim JS. The effect of hypercholesterolemia on rotator cuff disease. Clin Orthop Relat Res 2010;468:1493-7.

58 Longo UG, Franceschi F, Spiezia F, et al. Triglycerides and total serum cholesterol in rotator cuff tears: do they matter? Br J Sports Med 2010;44:948-51.

59 Welsh P, Woodward M, Rumley A, et al. Associations of plasma pro-inflammatory cytokines, fibrinogen, viscosity and C-reactive protein with cardiovascular risk factors and social deprivation: the fourth glasgow monica study. Br J Haematol 2008;141:852-61.

60 Moher D, Shamseer L, Clarke M, et al. Preferred reporting items for systematic review and meta-analysis protocols (PRISMA-P) 2015 statement. Syst Rev 2015;4:1

61 Howick J, Chalmers I, Glasziou P. The Oxford CEBM levels of evidence (introductory document); 2011.

62 Wells G, Shea B, O'Connell D, et al. The Newcastle-Ottawa scale (NOS) for assessing the quality of Non-Randomized studies in meta-analysis 2000. 
63 Higgins J, Green S. Cochrane Handbook for systematic reviews of interventions version 5.1.0, 2011.

64 Hallajzadeh J, Khoramdad M, Izadi N, et al. The association between metabolic syndrome and its components with systemic lupus erythematosus: a comprehensive systematic review and meta-analysis of observational studies. Lupus 2018;27:899-912.

65 Cohen J. A coefficient of agreement for nominal scales. Educ Psychol Meas 1960;20:37-46.

66 Applegate KA, Thiese MS, Merryweather AS, et al. Association between cardiovascular disease risk factors and rotator cuff tendinopathy: a cross-sectional study. J Occup Environ Med 2017;59:154-60.

67 Djerbi I, Chammas M, Mirous M-P, et al. Impact of cardiovascular risk factor on the prevalence and severity of symptomatic full-thickness rotator cuff tears. Orthop Traumatol Surg Res 2015;101:S269-73.

68 Juge P-A, Berard L, Kotti S, et al. Cardiometabolic risk factors in primary centred and rotator cuff-related shoulder osteoarthritis: a comparative study. RMD Open 2017;3:e000429.

69 Viera AJ, Garrett JM. Understanding interobserver agreement: the kappa statistic. Fam Med 2005;37:360-3.

70 Organisation WH. The ICD-10 classification of mental and behavioural disorders: clinical descriptions and diagnostic guidelines, 1992.

71 D'Agostino RB, Vasan RS, Pencina MJ, et al. General cardiovascular risk profile for use in primary care: the Framingham heart study. Circulation 2008;117:743-53.

72 Alexander MJL, Butcher JE, MacDonald PB. Effect of water exercise program on walking gait, flexibility, strength, self-reported disability and other psycho-social measures of older individuals with arthritis. Physiotherapy Canada 2001;53:203-11.

73 Gumina S, Arceri V, Carbone S, et al. The association between arterial hypertension and rotator cuff tear: the influence on rotator cuff tear sizes. J Shoulder Elbow Surg 2013;22:229-32.

74 Lin TT-L, Lin C-H, Chang C-L, et al. The effect of diabetes, hyperlipidemia, and statins on the development of rotator cuff disease: a nationwide, 11-year, longitudinal, population-based follow-up study. Am J Sports Med 2015;43:2126-32.

75 Pico-Espinosa OJ, Skillgate E, Tettamanti G, et al. Diabetes mellitus and hyperlipidaemia as risk factors for frequent pain in the back, neck and/or shoulders/arms among adults in Stockholm 2006 to 2010 - Results from the Stockholm Public Health Cohort. Scand J Pain 2017;15:1-7.

76 Titchener AG, White JJE, Hinchliffe SR, et al. Comorbidities in rotator cuff disease: a case-control study. J Shoulder Elbow Surg 2014;23:1282-8.

77 Chung SW, Choi BM, Kim JY, et al. Altered gene and protein expressions in torn rotator cuff tendon tissues in diabetic patients. Arthroscopy 2017;33:518-26.

78 Blaine TA, Kim Y-S, Voloshin I, et al. The molecular pathophysiology of subacromial bursitis in rotator cuff disease. J Shoulder Elbow Surg 2005;14:S84-9.

79 Voloshin I, Gelinas J, Maloney MD, et al. Proinflammatory cytokines and metalloproteases are expressed in the subacromial bursa in patients with rotator cuff disease. Arthroscopy 2005;21:1076. e1-1076.e9.

80 Dean BJF, Franklin SL, Carr AJ. A systematic review of the histological and molecular changes in rotator cuff disease. Bone Joint Res 2012;1:158-66.

81 Aroor AR, McKarns S, Demarco VG, et al. Maladaptive immune and inflammatory pathways lead to cardiovascular insulin resistance. Metabolism 2013;62:1543-52.

82 Deng Y, Scherer PE. Adipokines as novel biomarkers and regulators of the metabolic syndrome. Ann N Y Acad Sci 2010;1212:E1-19.
83 Al-Suhaimi EA, Shehzad A, Leptin SA. Leptin, resistin and visfatin: the missing link between endocrine metabolic disorders and immunity. Eur J Med Res 2013;18:12.

84 Fessel G, Li Y, Diederich V, et al. Advanced glycation end-products reduce collagen molecular sliding to affect collagen fibril damage mechanisms but not stiffness. PLoS One 2014;9:e110948.

85 Burner T, Gohr C, Mitton-Fitzgerald E, et al. Hyperglycemia reduces proteoglycan levels in tendons. Connect Tissue Res 2012;53:535-41.

86 Abella V, Scotece M, Conde J, et al. Adipokines, metabolic syndrome and rheumatic diseases. Journal of Immunology Research 2014;2014:1-14.

87 Lago F, Dieguez C, Gómez-Reino J, et al. The emerging role of adipokines as mediators of inflammation and immune responses. Cytokine Growth Factor Rev 2007:18:313-25.

88 Gandhi R, Perruccio AV, Rizek R, et al. Obesity-Related adipokines predict patient-reported shoulder pain. Obes Facts 2013;6:536-41.

89 Krysiak R, Handzlik-Orlik G, Okopien B. The role of adipokines in connective tissue diseases. Eur J Nutr 2012;51:513-28.

90 Abate M, Schiavone C, Salini V. Sonographic evaluation of the shoulder in asymptomatic elderly subjects with diabetes. BMC Musculoskelet Disord 2010;11:278.

91 Gaida JE, Alfredson H, Scott A, et al. Apolipoprotein A1 distribution pattern in the human Achilles tendon. Scand J Med Sci Sports 2018;28:1506-13.

92 von Bahr S, Movin T, Papadogiannakis N, et al. Mechanism of accumulation of cholesterol and cholestanol in tendons and the role of sterol 27-hydroxylase (CYP27A1). Arterioscler Thromb Vasc Biol 2002;22:1129-35.

93 Crouse JR, Grundy SM, Ahrens EH. Cholesterol distribution in the bulk tissues of man: variation with age. $J$ Clin Invest 1972;51:1292-6.

94 Finlayson R, Woods SJ. Lipid in the Achilles' tendon: a comparative study. Atherosclerosis 1975;21:371-89.

95 Adams CW, Bayliss OB, Baker RW, et al. Lipid deposits in ageing human arteries, tendons and fascia. Atherosclerosis 1974:19:429-40.

96 Halperin RO, Sesso HD, Ma J, et al. Dyslipidemia and the risk of incident hypertension in men. Hypertension 2006;47:45-50.

97 Tilley BJ, Cook JL, Docking SI, et al. Is higher serum cholesterol associated with altered tendon structure or tendon pain? A systematic review. Br J Sports Med 2015;49:1504-9.

98 McMaster WG, Kirabo A, Madhur MS, et al. Inflammation, immunity, and hypertensive end-organ damage. Circ Res 2015;116:1022-33.

99 Reuben DB, Cheh Al, Harris TB, et al. Peripheral blood markers of inflammation predict mortality and functional decline in highfunctioning community-dwelling older persons. J Am Geriatr Soc 2002;50:638-44.

100 Gold JE, Hallman DM, Hellström F, et al. Systematic review of biochemical biomarkers for neck and upper-extremity musculoskeletal disorders. Scand J Work Environ Health 2016:42:103-24.

101 Littlewood C, May S, Walters S. Epidemiology of rotator cuff tendinopathy: a systematic review. Shoulder Elbow 2013;5:256-65.

102 Bachasson D, Singh A, Shah SB, et al. The role of the peripheral and central nervous systems in rotator cuff disease. J Shoulder Elbow Surg 2015;24:1322-35.

103 Chester R, Jerosch-Herold C, Lewis J, et al. Psychological factors are associated with the outcome of physiotherapy for people with shoulder pain: a multicentre longitudinal cohort study. Br J Sports Med 2018;52:269-75.

104 Popay J, Roberts H, Sowden A, et al. Guidance on the conduct of narrative synthesis in systematic reviews: a product from the ESRC methods programme 2006. 